\title{
Supplementary Data
}

Perturbing phytoplankton: A tale of isotopic fractionation in two coccolithophore species

R. E. M. Rickaby ${ }^{1^{*}}$, J. Henderiks ${ }^{2^{*}}$, J. N. Young ${ }^{1}$

[1] \{ Department of Earth Sciences, Oxford University, Parks Road, Oxford, OX1 3PR \}

[2] \{ Department of Geology and Geochemistry, Stockholm University, Sweden; now at Department of Earth Sciences, Paleobiology Program, Uppsala University, Villavägen 16, 75236 Uppsala, Sweden\}

$\left[{ }^{*}\right]\{$ These authors contributed equally to this work\}

Correspondence to: R. E. M. Rickaby (rosr@earth.ox.ac.uk) 


\begin{tabular}{|c|c|c|c|c|c|c|c|c|c|c|c|}
\hline Treatment & Treatment & $\begin{array}{l}\text { AVG HCO3- } \\
(\mu \mathrm{mol} \mathrm{Kg-1]}\end{array}$ & $\begin{array}{l}\text { AVG CO32- } \\
(\mu \mathrm{mol} \mathrm{Kg-1]}\end{array}$ & 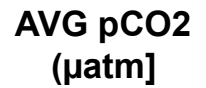 & $\begin{array}{c}\text { AVG } \mathrm{CO} 2(\mu \mathrm{mol} \\
\mathrm{kg}-1]\end{array}$ & $\begin{array}{c}\text { AVG pH } \\
\text { (NBS) }\end{array}$ & $\begin{array}{c}\text { AVG } \\
\text { Omega }\end{array}$ & $\begin{array}{l}\text { TPC raw } \\
\mu g\end{array}$ & $\begin{array}{l}\text { POC raw } \\
\mu \mathrm{g}\end{array}$ & $\begin{array}{l}13 C- \\
\text { TPC }\end{array}$ & 13C-POC \\
\hline \multirow[t]{2}{*}{$200 A$} & 200 & 1010.30 & 83.60 & 248.00 & 8.60 & 8.14 & 2.04 & 299.9 & 154.9 & -18.6 & -25.8 \\
\hline & 200 & 1010.30 & 83.60 & 248.00 & 8.60 & 8.14 & 2.04 & 298.1 & 149.7 & -18.5 & -26.3 \\
\hline \multirow[t]{2}{*}{ 200B } & 200 & 1087.75 & 55.15 & 421.25 & 14.60 & 7.93 & 1.35 & 295.0 & 180.6 & -19.0 & -25.0 \\
\hline & 200 & 1087.75 & 55.15 & 421.25 & 14.60 & 7.93 & 1.35 & 292.5 & 167.0 & -18.5 & -25.4 \\
\hline \multirow[t]{2}{*}{ 280A } & 280 & 1425.25 & 117.90 & 327.15 & 11.40 & 8.15 & 2.88 & 283.1 & 133.7 & -18.6 & -26.4 \\
\hline & 280 & 1425.25 & 117.90 & 327.15 & 11.40 & 8.15 & 2.88 & 279.8 & 159.2 & -18.7 & -25.6 \\
\hline \multirow[t]{2}{*}{ 280B } & 280 & 1445.20 & 105.45 & 371.00 & 12.75 & 8.10 & 2.57 & 420.8 & 263.7 & -18.7 & -23.3 \\
\hline & 280 & 1445.20 & 105.45 & 371.00 & 12.75 & 8.10 & 2.57 & 413.7 & 217.5 & -18.5 & -21.9 \\
\hline \multirow[t]{2}{*}{$380 \mathrm{~A}$} & 380 & 1908.10 & 153.25 & 450.90 & 15.65 & 8.14 & 3.74 & 450.7 & 288.3 & -18.5 & -22.9 \\
\hline & 380 & 1908.10 & 153.25 & 450.90 & 15.65 & 8.14 & 3.74 & 449.3 & 269.5 & -18.4 & -23.1 \\
\hline \multirow[t]{2}{*}{$380 B$} & 380 & 1939.15 & 141.55 & 497.80 & 17.30 & 8.10 & 3.45 & 356.5 & 220.0 & -18.3 & -23.2 \\
\hline & 380 & 1939.15 & 141.55 & 497.80 & 17.30 & 8.10 & 3.45 & 355.5 & 224.5 & -17.9 & -23.6 \\
\hline \multirow[t]{2}{*}{$950 A$} & 950 & 4889.95 & 353.70 & 1126.00 & 44.35 & 8.09 & 8.63 & 472.6 & 347.9 & -18.6 & -22.5 \\
\hline & 950 & 4889.95 & 353.70 & 1126.00 & 44.35 & 8.09 & 8.63 & 473.0 & 312.5 & -18.5 & -22.6 \\
\hline \multirow[t]{2}{*}{ 950B } & 950 & 4878.60 & 361.40 & 1237.50 & 43.00 & 8.10 & 8.82 & 470.7 & 239.9 & -18.8 & -23.6 \\
\hline & 950 & 4878.60 & 361.40 & 1237.50 & 43.00 & 8.10 & 8.82 & 456.4 & 359.3 & -18.4 & -22.1 \\
\hline \multirow[t]{2}{*}{$1400 \mathrm{~A}$} & 1400 & 7183.55 & 486.70 & 1993.90 & 69.25 & 8.06 & 11.87 & 452.2 & 291.2 & -18.2 & -22.6 \\
\hline & 1400 & 7183.55 & 486.70 & 1993.90 & 69.25 & 8.06 & 11.87 & 444.9 & 206.9 & -18.5 & -29.5 \\
\hline \multirow[t]{2}{*}{ 1400B } & 1400 & 7159.20 & 503.55 & 1907.45 & 66.25 & 8.08 & 12.28 & 445.2 & 263.4 & -18.9 & -23.2 \\
\hline & 1400 & 7159.20 & 503.55 & 1907.45 & 66.25 & 8.08 & 12.28 & 449.2 & 282.7 & -18.6 & -23.2 \\
\hline
\end{tabular}

Gephyrocapsa oceanica TPC, POC, POC, stable carbon isotopes, PON 
PONtpc PONpoc raw Blank TPC Blank POC Blank PON

\section{raw $\mu \mathrm{g} \quad \mu \mathrm{g}$}

21.2

21.1

19.8

20.8

20.4

20.2

27.6

27.3
28.0

28.0

28.0

24.0

23.2

30.8

30.6

30.8

30.0

29.5

29.4

28.6

28.9 $\mu \mathrm{g}$

$\begin{array}{lll} & \boldsymbol{\mu g} & \boldsymbol{\mu g} \\ 18.5 & 3.2 & \\ 18.5 & 3.0 & \\ 20.8 & 2.2 & \\ 19.7 & 1.9 & \\ 16.8 & & 1.3 \\ 18.8 & & 1.7 \\ 24.6 & & 2.9 \\ 21.5 & & 3.0 \\ 25.1 & & \\ 24.1 & 2.6 & \\ 22.4 & & \\ 22.3 & & \end{array}$

$\mu \mathrm{g}$

$\begin{array}{crr} & \text { TPC } \boldsymbol{\mu g} & \text { POC } \mu \mathrm{g} \\ 2.3 & \mathbf{\mu g} \\ 2.4 & 297.3 & 152.7 \\ 0.4 & 295.5 & 147.5 \\ 0.4 & 289.4 & 178.4 \\ 0.6 & 280.5 & 164.8 \\ 0.5 & 277.2 & 131.5 \\ 2.6 & 418.2 & 261.5 \\ 2.7 & 411.1 & 215.3 \\ & 448.1 & 286.1 \\ 1.4 & 446.7 & 267.3 \\ 1.6 & 353.9 & 217.8 \\ & 352.9 & 222.3 \\ & 470.0 & 345.7 \\ & 470.4 & 310.3 \\ & 468.1 & 237.7 \\ & 453.8 & 357.1 \\ & 449.6 & 289.0 \\ & 442.3 & 204.7 \\ & 442.6 & 261.2 \\ & 446.6 & 280.5\end{array}$

PONtpc PONpoc

19.8
19.7
18.4
19.4
19.0
18.8
26.2
25.9
26.6
26.6
22.6
21.8
29.4
29.2
29.4
28.6
28.1
28.0
27.2
27.5

18.1

23.0

23.5

22.5

20.8

20.7

28.7

25.0

21.6

24.2

24.8
PONtpc pg

TPC pg ml-1 POC pg ml-1 ml-1

$\begin{array}{rrrr}849500.0 & 436214.3 & 56642.9 & 48285.7 \\ 844357.1 & 421357.1 & 56357.1 & 48285.7 \\ 835500.0 & 509642.9 & 52642.9 & 54857.1 \\ 828357.1 & 470785.7 & 55500.0 & 51714.3 \\ 801500.0 & 375642.9 & 54357.1 & 43428.6 \\ 792071.4 & 448500.0 & 53785.7 & 49142.9 \\ 1194928.6 & 747071.4 & 74928.6 & 65714.3 \\ 1174642.9 & 615071.4 & 74071.4 & 56857.1 \\ 1280357.1 & 817357.1 & 76071.4 & 67142.9 \\ 1276357.1 & 763642.9 & 76071.4 & 64285.7 \\ 1011214.3 & 622214.3 & 64642.9 & 59428.6 \\ 1008357.1 & 635071.4 & 62357.1 & 59142.9 \\ 1342928.6 & 987642.9 & 84071.4 & 82000.0 \\ 1344071.4 & 886500.0 & 83500.0 & 71428.6 \\ 1337500.0 & 679071.4 & 84071.4 & 61714.3 \\ 1296642.9 & 1020214.3 & 81785.7 & 80000.0 \\ 1284642.9 & 825642.9 & 80357.1 & 69142.9 \\ 1263785.7 & 584785.7 & 80071.4 & 70857.1 \\ 1264642.9 & 746214.3 & 77785.7 & 61142.9 \\ 1276071.4 & 801357.1 & 78642.9 & 70000.0\end{array}$

PONpoc pg

19.2

15.2

17.2

19.9

28.0

21.4
24.5

1276071.4

801357.1

78642.9

70000.0 


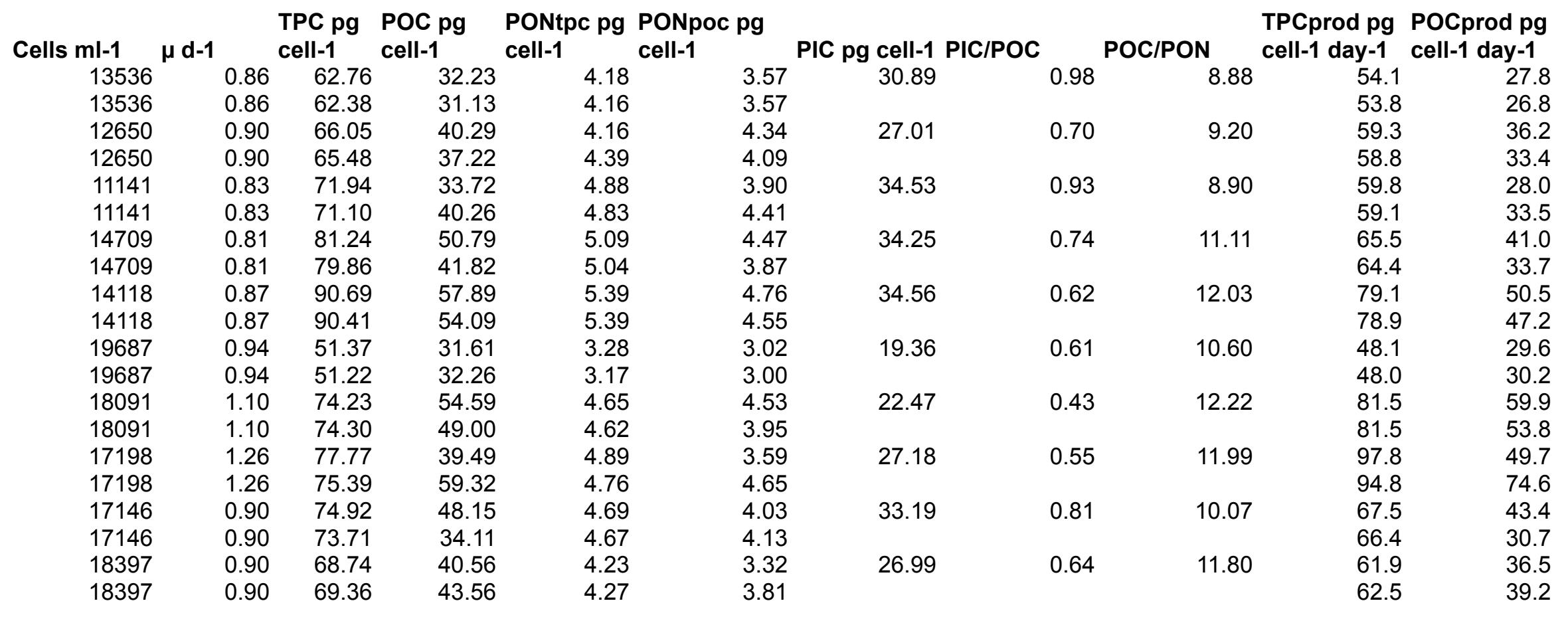


PONtpcprod PONpocpro

pg cell-1 day. $d$ pg cell-1

1 day-1
PICprod pg

cell-1 day-1 d13C-DIC to

$6^{\text {day-1 }} 3.1$

$3.6 \quad 3.1$

$\begin{array}{ll}3.7 & 3.9\end{array}$

$3.9 \quad 3.7$

$\begin{array}{ll}4.1 & 3.2 \\ 4.0 & 3.7\end{array}$

$4.1 \quad 3.6$

$4.1 \quad 3.1$

$4.7 \quad 4.1$

$4.7 \quad 4.0$

$3.1 \quad 2.8$

$5.1 \quad 5.0$

$5.1 \quad 4.3$

$6.1 \quad 4.5$

$6.0 \quad 5.9$

$4.2 \quad 3.6$

$\begin{array}{ll}3.8 & 3.0\end{array}$

3.9

3.4
d13C-DIC d13C-CO2

tfin $(0 / 00)$

\section{ep_CO2}

$-16.7 \quad 9.3$

$\begin{array}{ll}-16.7 & 9.8\end{array}$

$\begin{array}{ll}-16.7 & 9.8 \\ -16.7 & 8.5\end{array}$

$-16.7 \quad 8.9$

$\begin{array}{ll}-17.0 & 9.7\end{array}$

$\begin{array}{ll}-17.0 & 8.9\end{array}$

$-16.8 \quad 6.7$

$\begin{array}{ll}-16.8 & 5.2\end{array}$

$-16.7 \quad 6.3$

$-16.7 \quad 6.5$

$\begin{array}{ll}-16.7 & 6.6\end{array}$

$\begin{array}{ll}-16.7 & 7.0\end{array}$

$-16.9 \quad 5.7$

$\begin{array}{ll}-16.9 & 5.8\end{array}$

$-16.9-6.8$

$-16.9 \quad 5.3$

$-17.0 \quad 5.8$

$\begin{array}{rrrrr} & & & -17.0 & 12.9 \\ 24.3 & -7.10 & -6.90 & -16.9 & 6.4\end{array}$

$\begin{array}{ll}-16.9 & 6.4\end{array}$ 
AVG HCO3- AVG CO32- AVG pCO2 AVG CO2 AVG pH

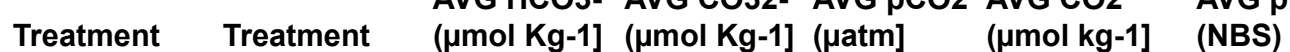

AVG Omega TPC raw

200A

$\begin{array}{rrr}1016.15 & 77.30 & 261.95 \\ 1016.15 & 77.30 & 261.95\end{array}$

$9.10 \quad 8.11$

261.95

420.15

9.10

1016.15

53.55

420.15

1066.40

53.55

335.80

14.60

280A

1429.45

114.90

335.80

280B

1429.45

114.90

364.10

1442.80

107.10

364.10

1934.65

141.05

497.25

1934.65

141.05

497.25

1942.80

131.45

539.65

$1942.80 \quad 131.45 \quad 539.65$

950A

4906.75

353.55

1286.30

4906.75

$353.55 \quad 1286.30$

$375.60 \quad 1189.50$

950B

4886.55

$375.60 \quad 1189.50$

1400A

$\begin{array}{rr}950 & 4886.55 \\ 1400 & 7167.55\end{array}$

$515.05 \quad 1868.10$

$1400 \quad 7167.55$

$515.05 \quad 1868.10$

1400B

$1400 \quad 7168.25$

526.75

1827.55

1827.55

14.60

11.70

11.70

12.65

12.65

17.30

17.30

18.75

18.75

44.70

44.70

41.30

41.30

64.90

64.90

63.45

63.45

8.11
8.11
7.92
7.92
8.14
8.14
8.10
8.10
8.10
8.10
8.06
8.06
8.09
8.09
8.12
8.12
8.09
8.09
8.10
8.10

1.89

361.3

362.2

437.0

165.7

Red $=10$ or more \% difference between duplicates

Blue=remarks in labbook

Red $^{*}=$ excluded

Coccolithus braarudii TPC, POC, POC, stable carbon isotopes, PON

Growth rates (coulter counter (CC) and manual microscopy counts)

$\%$ collapsed spheres and \%malformation 
PONpoc Blank TPC Blank POC Blank PON

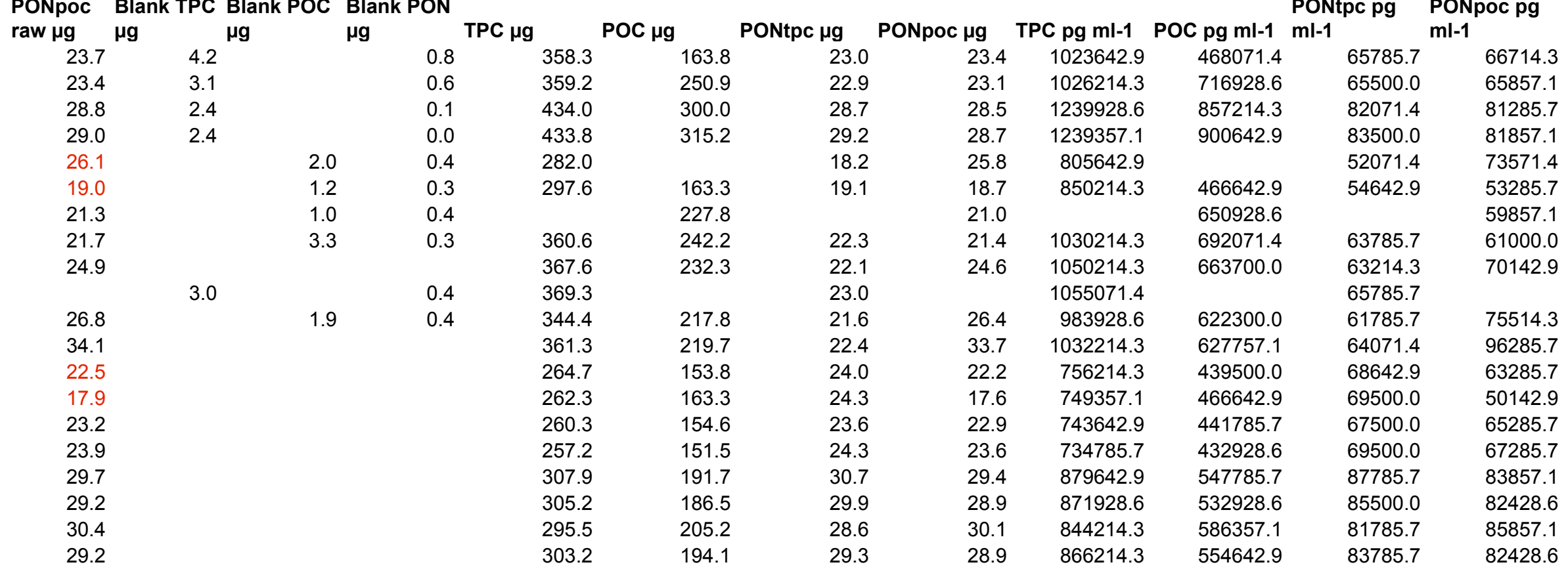


Cells ml-1

(as counted

by CC)

$\begin{array}{rrr}\mu \mathrm{d}-1 \text { (CC) } & \begin{array}{l}\text { Cells ml-1 } \\ \text { (LM counts) }\end{array} & \mu \text { d-1 (L } \\ \text { counts) }\end{array}$

TPC pg POC p

PONtpc pg PONpoc pg PIC pg

cell-1 cell-1

cell-1

PIC/POC POC/PON POC/PONtpc TPCprod pg

1803

1803

$0.62 \quad 2355$

$\begin{array}{lll}0.67 & 434.67 & 198.76\end{array}$

27.93

cell-1

$\begin{array}{crrr}\text { PIC/POC } & \text { POC/PON } & \text { POC/PONtpc } & \text { cell-1 day-1 } \\ 0.73 & 8.94 & 9.03 & 292.4\end{array}$

2106

0.57

$0.67 \quad 435.76$

304.43

27.81

$28.33 \quad 183.62$

292.4

2106

$\begin{array}{llll}3170 & 0.67 & 391.14 & 270.41\end{array}$

25.89

$25.64 \quad 113.79$

$0.41 \quad 10.77$

10.62

263.5

1257
1257

0.61

1540

$0.67 \quad 390.96$

284.11

26.34

25.82

1545

$0.61 \quad 1540$

$0.60 \quad 2253$

1545

$0.64 \quad 552.09$

303.01

33.81

$47.77 \quad 234.60$

0.77

7.36

8.75

263.3

2103

0.60

2253

0.64

0.642566

2103

1893

$0.64 \quad 2566$

35.48

34.60

$26.57 \quad 159.22$

0.53

11.11

$0.62 \quad 2120$

$\begin{array}{lll}0.67 & 409.28 & 258.65\end{array}$

28.31

27.08

24.64

25.64

$0.66 \quad 464.12$

293.54

$27.34 \quad 151.58$

0.59

9.46

10.53

1893

1511

1511

0.49

2120

$0.66 \quad 486.89$

296.11

29.14

$35.62 \quad 180.68$

0.61

7.28

10.29

294.2

$\begin{array}{ll}0.49 & 877 \\ 0.49 & 878\end{array}$

$\begin{array}{ll}0.47 & 862.27\end{array}$

501.14

30.22

1597

0.49

948

$0.47 \quad 854.46$

532.09

79.25

45.42

$72.16 \quad 341.75$

0.66

7.99

9.93

274.5

1597

0.49

948

$0.41 \quad 775.09$

456.68

71.20

2059

1657

0.51
0.57

0.57

667
667

$0.27 \quad 1265.69$

879.10

73.31

$68.87 \quad 318.41$

0.69

6.60

6.56

306.7

1657

667

$0.27 \quad 1298.67$

831.55

122.62

$128.72 \quad 426.86$

0.50

6.78

6.89 
POCprod pg PONtpcprod PONpocprod PICprod pg d13C-DIC to d13C-DIC tfin d13C-CO2 cell-1 day-1 pg cell-1 day-1 pg cell-1 day-1 cell-1 day-1 (\%o)

$\begin{array}{lllll}133.7 & 18.8 & 19.1 & 123.5 & -7.25 \\ 204.8 & 18.7 & 18.8 & & \\ 182.1 & 17.4 & 17.3 & 76.6 & -7.25 \\ 191.4 & 17.7 & 17.4 & & \\ & 21.7 & 30.7 & 150.9 & -7.18 \\ 194.9 & 22.8 & 22.3 & & \\ 185.9 & & 17.1 & 102.4 & -7.18 \\ 197.6 & 18.2 & 17.4 & & \\ 173.5 & 16.5 & 18.3 & 101.7 & -7.22 \\ & 17.2 & & & \\ 194.0 & 19.3 & 23.5 & 119.4 & -7.22 \\ 195.7 & 20.0 & 30.0 & & \\ 237.8 & 37.1 & 34.2 & 162.2 & -7.15 \\ 252.5 & 37.6 & 27.1 & & \\ 190.3 & 29.1 & 28.1 & 130.1 & -7.15 \\ 186.5 & 29.9 & 29.0 & & \\ & & & & -7.10 \\ 241.4 & 33.7 & 35.3 & 117.2 & -7.10 \\ 228.3 & 34.5 & 33.9 & & \end{array}$

(\%) (\%o)

$-6.49$

$-6.55$

$-6.89$

$-6.81$

$-$

$-6.70$

$-6.95$

$-6.97$

$-6.95$

$-7.53$ 4 $-18.4$ $-18.4$

$-18.4$

$-18.4$

$-18.8$

$-18.8$

$-18.7$

$-18.7$

$-18.6$

-18.6
-18.6

$-18.8$

$-18.8$

$-18.8$

$-18.8$

$-18.8$

$-18.8$

$-19.4$

$-19.4$ morphologies O2 sphers spheres spheres (malformation) $\begin{array}{lllll}7.0 & 14.1 & 269 & 44 & 10.2\end{array}$

2.7

2.5
2.5

$24.5 \quad 234 \quad 76$

$\begin{array}{lll}18.0 & 250 & 55\end{array}$

12.4

3.6

2.8
2.4

$19.0 \quad 247$

4.6

$21.6 \quad 240$

66

17.4

4.6

6.9

$16.8 \quad 272$

55

16.0

79.5

82.9

98.1

95.1

63

245

76.1

74.4

8.0

7.0

7.2 